\title{
TRIM27 contributes to glomerular endothelial cell injury in lupus nephritis by mediating the Fox01 signaling pathway
}

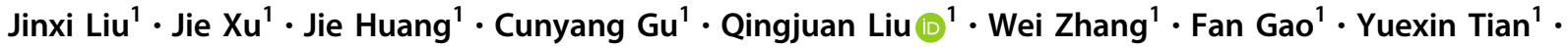 \\ Xinyan Miao ${ }^{1} \cdot$ Zixuan Zhu $^{2} \cdot$ Baiyun $\mathrm{Jia}^{2} \cdot \mathrm{Yu}$ Tian $^{1,3} \cdot$ Lunbi Wu$^{1} \cdot$ Hang Zhao ${ }^{1} \cdot$ Xiaojuan Feng $\mathbb{D}^{1} \cdot$ Shuxia Liu $\mathbb{D}^{1}$
}

Received: 4 September 2020 / Revised: 24 March 2021 / Accepted: 25 March 2021 / Published online: 14 April 2021

(c) The Author(s), under exclusive licence to United States and Canadian Academy of Pathology 2021, corrected publication 2021

\begin{abstract}
Tripartite motif-containing 27 (TRIM27) belongs to the triple motif (TRIM) protein family, which plays a role in a variety of biological activities. Our previous study showed that the TRIM27 protein was highly expressed in the glomerular endothelial cells of patients suffering from lupus nephritis (LN). However, whether TRIM27 is involved in the injury of glomerular endothelial cells in lupus nephritis remains to be clarified. Here, we detected the expression of the TRIM27 protein in glomerular endothelial cells in vivo and in vitro. In addition, the influence of TRIM27 knockdown on endothelial cell damage in MRL/lpr mice and cultured human renal glomerular endothelial cells (HRGECs) was explored. The results revealed that the expression of TRIM27 in endothelial cells was significantly enhanced in vivo and in vitro. Downregulating the expression of TRIM27 inhibited the breakdown of the glycocalyx and the injury of endothelial cells via the FoxO1 pathway. Moreover, HRGECs transfected with the WT-FoxO1 plasmid showed a reduction in impairment caused by LN plasma. Furthermore, suppression of the protein kinase B (Akt) pathway could attenuate damage by mediating the expression of TRIM27. Thus, the present study showed that TRIM27 participated in the injury of glomerular endothelial cells and served as a potential therapeutic target for the treatment of lupus nephritis.
\end{abstract}

\section{Introduction}

Systemic lupus erythematosus (SLE) is an autoimmune disease that affects multiple systems and organs. Approximately

These authors contributed equally: Jinxi Liu, Jie Xu

Supplementary information The online version contains supplementary material available at https://doi.org/10.1038/s41374021-00591-9.

Xiaojuan Feng

fengxiaojuan1985@163.com

$\triangle$ Shuxia Liu

shuxialiu@hebmu.edu.cn

1 Department of Pathology; Center of Metabolic Diseases and Cancer Research, Institute of Medical and Health Science, Hebei Medical University; Key Laboratory of Kidney Diseases of Hebei Province, Shijiazhuang, China

2 Basic Medicine, Hebei Medical University, Shijiazhuang, China

3 Department of Rheumatology, The Second Affiliated Hospital of Hebei Medical University, Shijiazhuang, China
$40-60 \%$ of SLE patients develop lupus nephritis, which will gradually evolve into end-stage renal disease [1,2].

Proteinuria is one of the most typical clinical manifestations of lupus nephritis and is induced by the destruction of the glomerular filtration barrier (GFB) [3, 4]. The GFB consists of a three-layer structure composed of endothelial cells, basement membrane, and podocytes [5]. The glomerular endothelial cells have many fenestrae that are 70 to 100 nanometers in diameter and are covered by the carbohydrate-rich glycocalyx at the cell surface [6]. Previous research has discovered that there is a positive correlation between the loss of the glycocalyx and proteinuria in various animal models [7]. A recent study demonstrated that the impairment of endothelial cells partially contributed to the appearance of severe proteinuria in patients with lupus nephritis [8]. In our previous research, the shedding of the glycocalyx was also observed in lupus nephritis (LN) patients and was positively correlated with proteinuria. Therefore, it is critical to explore the mechanism of the degradation of the glycocalyx and glomerular endothelial cell damage in lupus nephritis.

TRIM27 is a member of the TRIM family and contains three conserved domains: a RING finger, one or two B-box 
motifs, and a coiled-coil region. TRIM family proteins also contain a highly variable C-terminal region [9]. Reportedly, TRIM27 is involved in the regulation of many cellular functions and biological processes, including tumorigenesis [10], innate immunity [11], and viral replication [12]. Our previous research proved that the high expression of TRIM27 promoted mesangial cell proliferation and extracellular matrix deposition via the FoxO1 signaling pathway in lupus nephritis [13]. In addition, the overexpression of TRIM27 was observed not only in mesangial cells but also in endothelial cells of patients with lupus nephritis. Therefore, whether TRIM27 participates in the breakdown of the glycocalyx and damage to glomerular endothelial cells needs to be investigated.

In summary, the purpose of this study was to explore the role and mechanism of TRIM27 in mediating glomerular endothelial cell injury in LN. Conclusively, the downregulation of TRIM27 could reduce the loss of the glycocalyx and injury to glomerular endothelial cells in MRL/lpr mice and cultured human glomerular endothelial cells stimulated with LN plasma. Moreover, pretreatment with the Akt pathway inhibitor LY294002 could reduce the expression of the TRIM27 protein and the dysfunction of glomerular endothelial cells via the FoxO1 pathway. Thus, the present study reveals that TRIM27 might be a target for the inhibition of the pathogenesis and the targeted therapy of lupus nephritis.

\section{Materials and methods}

\section{Patients}

The plasma samples were acquired from five patients who were diagnosed with lupus nephritis in the Inpatient Department of Nephrology at the Second Hospital of Hebei Medical University from 2018 to 2019. The same number of plasma samples was collected from sex- and age-matched healthy Chinese donors, which were used as the control group.

\section{Reagents}

Rabbit anti-TRIM27 and mouse anti-CD31 and antiGAPDH antibodies were purchased from Proteintech Group (Wuhan, Hubei, China). Rabbit anti-syndecan-1, anti-VCAM-1, anti-FoxO1, anti-Akt, and anti-p-Akt-Ser473 antibodies were purchased from Abcam (Cambridge, UK). Rabbit anti-p-FoxO1-Ser256 and anti-p-Akt-Thr308 antibodies were purchased from Cell Signaling Technology (Boston, MA, USA). Mouse anti-ubiquitin antibody and protein $A+G$ agarose beads were purchased from Santa Cruz Biotechnology, Inc (Dallas, Texas, USA). Phalloidin was purchased from US Everbright Inc (Suzhou, Jiangsu, China). LY294002 was purchased from MedChemExpress (NJ). SC79 was purchased from Apexbio (Houston, Texas, USA). Lipofectamine 3000 was purchased from Invitrogen (Carlsbad, CA, USA). Mouse syndecan-1 and VCAM-1 enzyme-linked immunosorbent assay (ELISA) kits were purchased from Shanghai Zcibio Technology Co., Ltd. Human syndecan-1 and VCAM-1 ELISA kits were purchased from Elisa Biotech Co., Ltd. The Total Nitric Oxide Assay Kit was purchased from Beyotime Biotechnology (Shanghai, Jiangsu, China).

\section{Cell culture and groups}

Human renal glomerular endothelial cells (HRGECs) were purchased from ScienCell (Carlsbad, CA, USA). HRGECs were cultured in $5 \% \mathrm{CO}_{2}$ in humidified air at $37^{\circ} \mathrm{C}$. The HRGECs were cultured in endothelial cell medium (ECM, ScienCell) supplemented with 5\% fetal bovine serum (FBS, ScienCell), $1 \%$ penicillin/streptomycin solution (P/S, ScienCell), and $1 \%$ endothelial cell growth supplement (ECGs, ScienCell).

The groups were generated as follows:

1. To investigate the effect of LN plasma on glomerular endothelial cells, HRGECs were randomly divided into two groups: the control group and the LN plasma group (LN group). HRGECs were exposed to $\mathrm{LN}$ plasma (5\%) collected at different times to detect the expression of TRIM27, VCAM-1, p-Akt-Ser473, p-Akt-Thr308, Akt, pFoxO1-Ser256 and FoxO1 protein.

2. To verify whether knocking down TRIM27 could reduce the damage to HRGECs in lupus nephritis, the HRGECs were randomly divided into four groups: the control group, $\mathrm{LN}$ group, $\mathrm{LN}+\mathrm{Sh}$-TRIM27 group and $\mathrm{LN}+\mathrm{NC}$ (LN + shramble sh-RNA) group. The expression of TRIM27, VCAM-1, p-Akt-Ser473, p-Akt-Thr308, Akt, p-FoxO1-Ser256 and FoxO1 protein were detected by western blot analysis in HRGECs after treatment with LN plasma. The cytoskeleton of HRGECs was observed by immunofluorescence staining (IF). The levels of syndecan1 and VCAM- 1 in the supernatant were measured by an enzyme-linked immunosorbent assay (ELISA). A total nitric oxide assay kit was used to detect the NO content.

3. To investigate how TRIM27 mediated the expression of FoxO1, HRGECs were randomly divided into groups: the control group, $\mathrm{LN}$ group, $\mathrm{LN}+\mathrm{Sh}$ TRIM27 group and $\mathrm{LN}+\mathrm{NC}(\mathrm{LN}+$ scramble shRNA) group or the control group, control+WTTRIM27 group, control+NC (LN + shramble RNA) separately. The mRNA and protein level of TRIM27, p-FoxO1-Ser256 and FoxO1 was detected in HRGECs. The ubiquitination level of FoxO1 was measured by co-immunoprecipitation. 
4. To further explore the influence of FoxO1 overexpression on glomerular endothelial cell injury, HRGECs were randomly divided into four groups: the control group, LN group, LN + pcDNA3.1-WTFoxO1 group and $\mathrm{LN}+\mathrm{NC}(\mathrm{LN}+$ shramble shRNA) group. After stimulation with $\mathrm{LN}$ plasma, HRGECs were collected, and the expression of pFoxO1-Ser256, FoxO1, and VCAM-1 protein were detected. The levels of syndecan-1, VCAM-1 and NO production were detected as described above.

5. In the PI3K/Akt signaling pathway inhibition experiment, the HRGECs were randomly divided into four groups: the control group, LN group, LN + LY294002 group, and LN + DMSO group. The cells in the $\mathrm{LN}+$ LY294002 group were pretreated with LY294002 (25 $\mu \mathrm{M})$ for $1 \mathrm{~h}$. After the HRGECs were collected, the protein levels of p-Akt-Ser473, p-Akt-Thr308, Akt, TRIM27, VCAM-1, p-FoxO1-Ser256, and FoxO1 were measured by western blot analysis. NO production and the levels of syndecan-1 and VCAM-1 in the medium were detected as described above.

6. In the PI3K/Akt signaling pathway activation experiment, the HRGECs were randomly divided into three groups: the control group, control+SC79 group, and control+DMSO group. The cells in the control+SC79 group were pretreated with SC79 $(4 \mu \mathrm{g} / \mathrm{mL})$ for $1 \mathrm{~h}$. Then the expression of p-Akt-Ser473, p-Akt-Thr308, Akt and TRIM27 protein were detected by western blot analysis.

\section{Plasmids and transfection}

The HRGECs were transfected with LV-U6> TRIM27PGK $>$ EGFP/T2-A/Puro or LV-U6 > Scramble-shRNAPGK $>$ EGFP/T2A/Puro (Cyagen Biotechnology, Suzhou, China) according to the manufacturer's protocols. The target sequence was GCAGCTCTATCACTCGTTGAACTCGAGTTCAACGAGTGATAGAGCTGC. The WT-TRIM 27 plasmid was purchased from Fulengen,Co. (Guangzhou, Guangdong, China). The pcDNA3.1-WT-FoxO1 plasmid was kindly provided by Prof. Hao. The HRGECs were transfected with the plasmids using Lipofectamine 3000 according to the manufacturer's protocols (Invitrogen, Carlsbad, CA).

\section{Animal studies}

Female MRL/lpr mice and MRL/MPJ mice were purchased from the Jackson Laboratory (000485). All experiments were approved by the Institutional Animal Care and Use Committee of Hebei Medical University (approval ID: HebMU 20080026). Fifteen 14-week-old female MRL/lpr mice were randomly divided into the MRL/lpr group, MRL/lpr+ShTRIM27 group, MRL/lpr+EC-shTRIM27 (MRL/lpr+ endothelial cells-shTRIM27, MRL/lpr+TIE-ShTRIM27) group and $\mathrm{MRL} / \mathrm{lpr}+\mathrm{NC}$ group, and five MRL/MPJ mice of the same age and gender were used as the control group. The mice in the MRL/lpr+sh-TRIM27, MRL/lpr+EC-shTRIM27 and MRL/ $\mathrm{lpr}+\mathrm{NC}$ groups were injected with $50 \mu \mathrm{L}$ containing $1.6 \times 10^{11}$ vector genome $(\mathrm{vg})$ infective units of recombinant adenoassociated virus (Hanbio Biotechnology Co., Ltd, Shanghai, China) in both renal cortices. Five MRL/MPJ mice were injected with $50 \mu \mathrm{L}$ normal saline. After 10 weeks, these mice were sacrificed, and urine, serum, and renal cortex samples were collected for subsequent detection.

\section{HE}

Take the kidney tissue and fix it in $4 \%$ paraformaldehyde solution. After the fixation was complete, the gradient ethanolxylene was dehydrated and transparent, fully immersed in wax and embedded, serially sectioned to $2-\mu \mathrm{m}$, deparaffinized after drying, and placed in hematoxylin-iridine stain in red solution, washed with water after dyeing, dehydrated with gradient ethanol, transparent xylene, and mount with neutral gum. After the gum is dried, place it under a microscope to observe the histopathological changes.

\section{PAS}

Paraffin sections were routinely dewaxed, washed with double distilled water, immersed in $0.5 \%$ periodic acid solution for $10 \mathrm{~min}$, and washed twice with double distilled water. Then, the slices were stained with Schiff reagent for $15 \mathrm{~min}$, washed with running water for $10 \mathrm{~min}$, restained with hematoxylin staining solution for $2 \mathrm{~min}$, differentiated with $1 \%$ hydrochloric acid ethanol, washed with running water for $10 \mathrm{~min}$, turned blue, dehydrated with conventional gradient alcohol, transparent with xylene and sealed with gum. At last, the sections were observed and taken pictures under light microscope.

\section{Immunohistochemistry (IHC)}

Kidney tissue was fixed with $4 \%$ paraformaldehyde and embedded in paraffin. Then, 4- $\mu$ m-thick sections were dewaxed in xylene and rehydrated with graded ethanol. The antigens were repaired using a pressure cooker and washed with $0.01 \mathrm{~mol} / \mathrm{L}$ phosphate-buffered saline (PBS). Endogenous peroxidase was blocked with $3 \% \mathrm{H}_{2} \mathrm{O}_{2}$ for 30 min at room temperature. The slides were blocked with $10 \%$ goat serum and then incubated with anti-TRIM27 (1:100), antiVCAM-1 (1:200), and anti-CD31 (1:500) antibodies at $4{ }^{\circ} \mathrm{C}$ overnight. The following day, the slides were incubated with HRP-conjugated anti-rabbit/mouse $\mathrm{IgG}$, which was 
followed by diaminobenzidine (DAB) staining. Finally, images were captured using an Olympus microscope (Olympus, BX71, Tokyo, Japan). The expression of stained proteins were characterized quantitatively by digital image analysis using Image Pro-Plus 5.0 software (Media Cybernetics, Silver Spring, MD).

\section{Total protein extraction and western blot analysis}

Renal cortex tissue from mice or HRGECs from different groups were placed in radioimmunoprecipitation assay (RIPA) buffer, lysed for $1 \mathrm{~h}$, and then centrifuged at $120,000 \mathrm{~g}$ at $4{ }^{\circ} \mathrm{C}$ for $20 \mathrm{~min}$. The supernatant was collected, and the relative levels of protein expression were detected by western blotting. The protein was separated by $10 \%$ sodium dodecyl sulfate-polyacrylamide gel electrophoresis (SDS-PAGE) and transferred to a polyvinylidene fluoride (PVDF) membrane (Millipore, Billerica, USA). The membrane was blocked with $5 \%$ bovine serum albumin (BSA) for $1.5 \mathrm{~h}$ at $37^{\circ} \mathrm{C}$ and then incubated with antiTRIM27 (1:1000), VCAM-1 (1:1000), p-Akt-Ser473 (1:2000), p-Akt-Thr308 (1:1000) FoxO1 (1:1000), and pFoxO1 (1:1000) antibodies at $4{ }^{\circ} \mathrm{C}$ overnight. Subsequently, the membrane was incubated with a goat anti-rabbit/mouse IgG secondary antibody (Proteintech, Wuhan, China) diluted 1:5000. The film was imaged using the LI-COR Odyssey Infrared Imaging System (Lincoln, NE, USA). All experiments were repeated at least three times.

\section{Immunofluorescence (IF)}

The HRGECs were inoculated in six-well plates, fixed with $4 \%$ paraformaldehyde for $30 \mathrm{~min}$ at room temperature, and lysed with $0.1 \%$ Triton X-100 for $15 \mathrm{~min}$ at room temperature. Then, the HRGECs or frozen sections were blocked with $10 \%$ goat serum at $37^{\circ} \mathrm{C}$ for $30 \mathrm{~min}$ and incubated with anti-TRIM27 (1:100), anti-syndecan-1 (1:200), and anti-CD31 (1:500) antibodies at $4{ }^{\circ} \mathrm{C}$ overnight. The next day, the cells or frozen sections were incubated with FITC-conjugated goat anti-rabbit IgG/ TRITC-conjugated goat anti-mouse IgG or phalloidin (1:50) at $37^{\circ} \mathrm{C}$ for $2 \mathrm{~h}$ followed by treatment with $4^{\prime}, 6$-diamidino2-phenylindole (DAPI; Southernbiotech, Birmingham). The sections were observed with an Olympus microscope (Olympus, Tokyo, Japan).

\section{ELISA}

The cell supernatants were collected from cultured HRGECs. The levels of SDC-1 and VCAM-1 in the supernatants or serum of mice were measured by ELISA kits according to the instructions. The absorbance was measured with a spectrophotometer at $450 \mathrm{~nm}$.

\section{NO measurements}

The HRGECs were lysed at $4{ }^{\circ} \mathrm{C}$ for $1 \mathrm{~h}$ to generate the nitric oxide lysate and then centrifuged at $120,000 \times g$ for $20 \mathrm{~min}$. The supernatants were collected, and the NO contents were detected by using the total nitric oxide assay kit in accordance with the manufacturer's instructions. The absorbance was measured with a spectrophotometer at 540 $\mathrm{nm}$ to calculate the total amount of NO.

\section{Real-time PCR}

The TRIZOL regent (Invitrogen) was employed to extract the total RNA from HRGECs. The complementary DNA (cDNA) was obtained by a reverse transcription kit. Reverse transcription-polymerase chain reaction (RT-PCR) was performed with FoxO1-specific primers (sense 5'-CAG CAA ATC AAG TTA TGG AGG A- $3^{\prime}$ and antisense $5^{\prime}$-TAT CAT TGT GGG GAG GAG AGT C-3') and SYBR Premix EX Taq 2 for 35 cycles $\left(95^{\circ} \mathrm{C}\right.$ for $30 \mathrm{~s}, 55^{\circ} \mathrm{C}$ for $30 \mathrm{~s}$ and $72{ }^{\circ} \mathrm{C}$ for $30 \mathrm{~s}$ ). GAPDH (sense $5^{\prime}$-CGC TGA GTA CGT CGT GGA GTC- $3^{\prime}$ and antisense 5'-GCT GAT GAT CTT GAG GCT GTT GTC-3') were amplified as internal control. The $2^{-\Delta \Delta C T}$ method was used to normalize the qPCR cDNAs. All experiments were repeated at least three times.

\section{CO-IP}

The total protein was extracted by cell lysis. $1 / 5$ of the total volume was used as the input control group of western blot. The remained cell lysates were incubated with anti-FoxO1 antibody at $4{ }^{\circ} \mathrm{C}$ overnight, then incubated with agarose beads protein $\mathrm{A}+\mathrm{G}$ overnight, centrifuged at $4{ }^{\circ} \mathrm{C} 500 \mathrm{~g}$, and the precipitates were suspended in washing buffer. The supernatant was detected by Western blot.

\section{Statistical analysis}

The quantitative data are shown as the mean \pm standard deviation (SD). The statistical significance was determined by one-way analysis of variance (ANOVA) or the Student-Newman-Keuls test. A $p$ value less than 0.05 was considered statistically significant.

\section{Results}

\section{Knockdown of TRIM27 suppressed the shedding of the glycocalyx and glomerular endothelial cell injury in MRL/lpr mice}

To investigate the effect of the TRIM27 protein on the dysfunction of glomerular endothelial cells, IF was used to 
explore the expression of TRIM27 protein in MRL/lpr mice. As demonstrated in Fig. 1a, the positive signal of TRIM27 mainly existed in the cytoplasm and nucleus of glomerular endothelial cells in mice, and it was enhanced significantly in LN mice compared with that in the control group.

Next, TRIM27-shRNA-AAV or NC-AAV was directly injected into the cortex of both kidneys of MRL/lpr mice in situ to achieve the effect of knocking down TRIM27. To confirm the efficiency of knockdown via TRIM27-shRNAAAV, western blotting was employed, and the results (Fig. 1b) showed that the protein level of TRIM27 was obviously increased in the kidneys of the MRL/lpr mice compared with that in the kidneys of the control mice; moreover, it was obviously decreased after injection with TRIM27-shRNA-AAV, whereas little change resulted from injection with NC-AAV.

Since syndecan-1 is a specific component of the glycocalyx, we applied IF staining to detect its expression. The results (Fig. 1c) indicated that in the control mice, the syndecan-1 protein was located in the cytoplasm and the membrane of glomerular cells, and it was reduced significantly in the LN mice. After knocking down the expression of TRIM27, syndecan-1 expression was partially recovered, indicating that TRIM27 mediated the loss of the glycocalyx in glomerular endothelial cells. As the syndecan-1 protein could be released by endothelial cells into the serum, ELISA analysis was applied to test the serum syndecan-1 level. As illustrated in Fig. 1d, the serum syndecan-1 level was remarkably higher in the LN group compared to that in the control group, and suppressing the expression of TRIM27 was able to decrease the syndecan-1 protein level in the serum. Therefore, the above results indicated the deterioration of the glycocalyx protective layer, which was an important sign of endothelial destruction.

In addition, previous research reported that the VCAM-1 protein was mainly expressed in activated endothelial cells, which initiated the injury of endothelial cells [14, 15]. IHC analysis results (Fig. 1e) revealed the positive staining of VCAM-1. In addition, little positive signal associated with VCAM-1 was found in the control mice, whereas the VCAM-1 signal was obviously enhanced in the MRL/lpr mice. The downregulation of TRIM27 could reduce the expression of VCAM-1. Western blot analysis results (Fig. 1b) also showed that the expression of VCAM-1 was significantly elevated in LN mice compared with that in control mice, whereas injection with TRIM27-shRNAAAV reduced the VCAM-1 level, which was consistent with the immunohistochemistry results. As expected, the VCAM-1 protein level (Fig. 1f) in the serum was dramatically higher in the LN mice compared to that in the control mice, while inhibition of TRIM27 could reduce the VCAM-1 protein level, which was consistent with previous results.
Furthermore, the level of NO in the kidney cortex of mice was detected, since increasing evidence has indicated that the enrichment of NO usually occurs in the early stages of ultrafiltration and GFR elevation [16, 17]. As shown in Fig. 1g, compared with that in the control group, the level of NO was evidently elevated in the LN group. In addition, TRIM27shRNA-AAV treatment abolished the increase in the NO level, whereas little change was observed in the NC-AAV group.

Finally, to specifically knockdown the expression of TRIM27 in glomerular endothelial cells, the TIE-TRIM27shRNA-AAV was injected to the kidney of MRL/lpr mice. As shown in Fig. 2a, b, the expression of TRIM27 in glomerular endothelial cells was downregulated effectively. The results (Fig. 2c) showed that UPro/24 hr was dramatically decreased in TIE-TRIM27-shRNA-AAV mice compared with MRL/lpr mice with little change of serum creatinine (Scr), and blood urea nitrogen (BUN). Expectively, the loss of glycocalyx and damage of endothelial cells was reduced after suppression of TRIM27 in MRL/lpr mice (Fig. 2d-i).

\section{Suppression of TRIM27 reduced the dysfunction of glomerular endothelial cells in vitro}

To imitate the microenvironment of $\mathrm{LN}$, plasma from LN patients was used to stimulate human glomerular endothelial cells. First, as illustrated in Fig. 3a, after treatment with LN plasma, the expression of TRIM27 protein was obviously increased in a time-dependent manner. In addition, the IF results (Fig. 3b) demonstrated that the positive signal of TRIM27, which was weak, was mainly distributed in the nuclei of HRGECs in the control group. After stimulation with LN plasma, the TRIM27 signal was dramatically enhanced and located mainly in the cytoplasm. The above results indicated that the expression of TRIM27 protein was increased in HRGECs after LN plasma treatment.

Afterward, to investigate whether overexpression of TRIM27 mediated the dysfunction of HRGECs, TRIM27shRNA-LV, or NC-LV was transfected into HRGECs. The western blotting results (Fig. 3c) revealed that the protein level of TRIM27 was clearly decreased after transfection with TRIM27-shRNA-LV, whereas little change was observed after transfection with NC-LV.

Next, degeneration of the glycocalyx layer was detected. As shown in Fig. 3d, the expression of the syndecan-1 protein mainly occurred in the membrane and cytoplasm of HRGECs. Compared with that in the control group, the positive signal of the syndecan-1 protein was significantly reduced in the LN group. Furthermore, TRIM27-shRNA-LV repressed the effect of LN plasma on syndecan-1 expression. ELISA (Fig. 3e) was applied to test the protein level of syndecan- 1 in the culture medium. The results demonstrated that the syndecan-1 


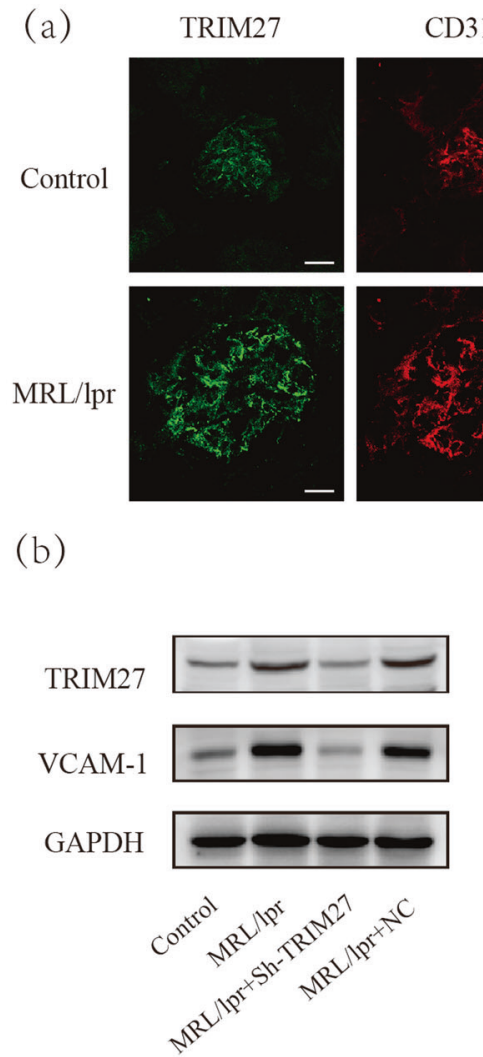

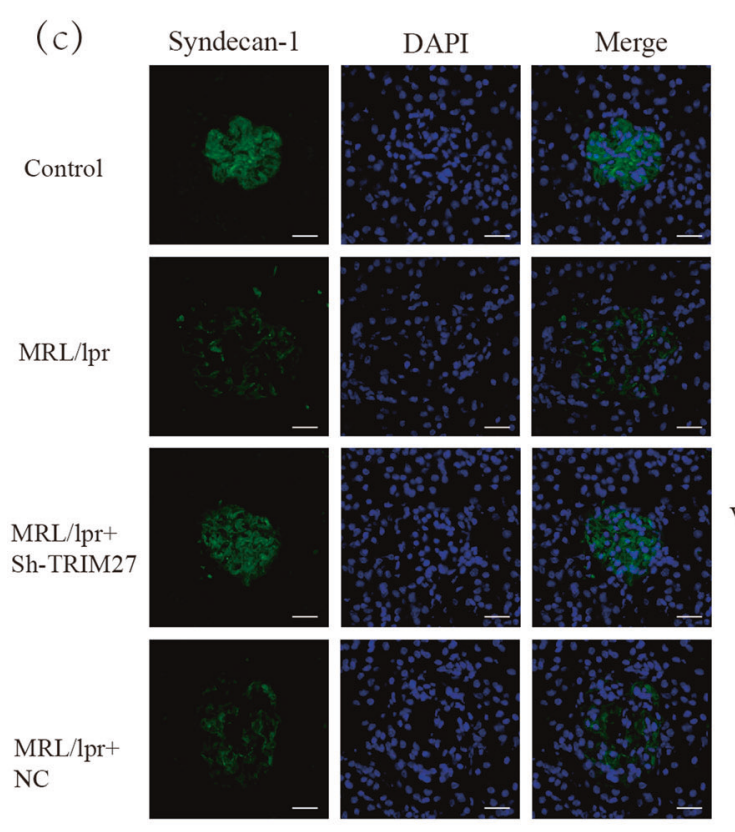

Fig. 1 TRIM27 mediated the degeneration of the glycocalyx and glomerular endothelial cell injury in MRL/lpr mice. a IF analysis showed that the expression of TRIM27 in glomerular endothelial cells of MRL/lpr mice was higher than that in glomerular endothelial cells of control mice. b The expression of TRIM27 and VCAM- 1 in the kidney cortex of mice was tested by western blotting. $\mathbf{c}$ The expression of syndecan-1 in the glomeruli of MRL/lpr mice was detected by IF after injection with TRIM27-shRNA-AAV or NC-AAV. d, f The ELISA results showed the content of syndecan- 1 and VCAM-1 in the (f)

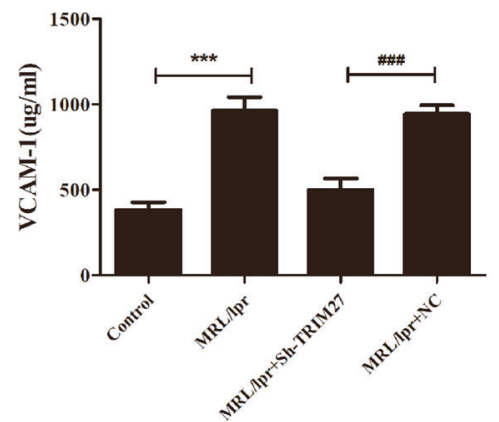

(g)

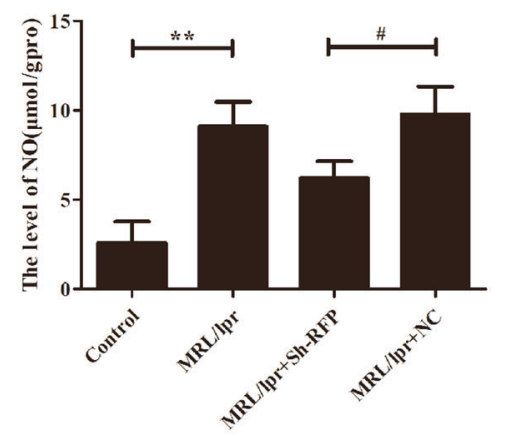

(d)

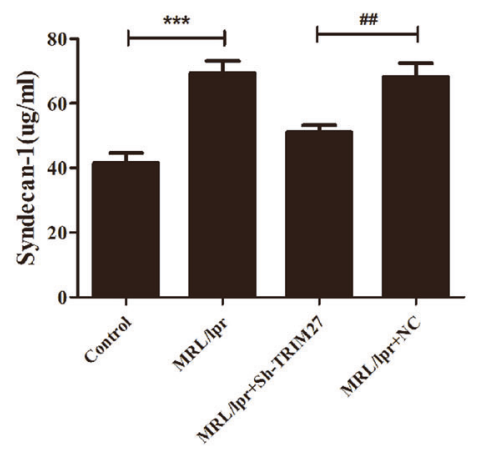

(e)

$\square \operatorname{TRIM} 27$

$\square$ VCAM-1
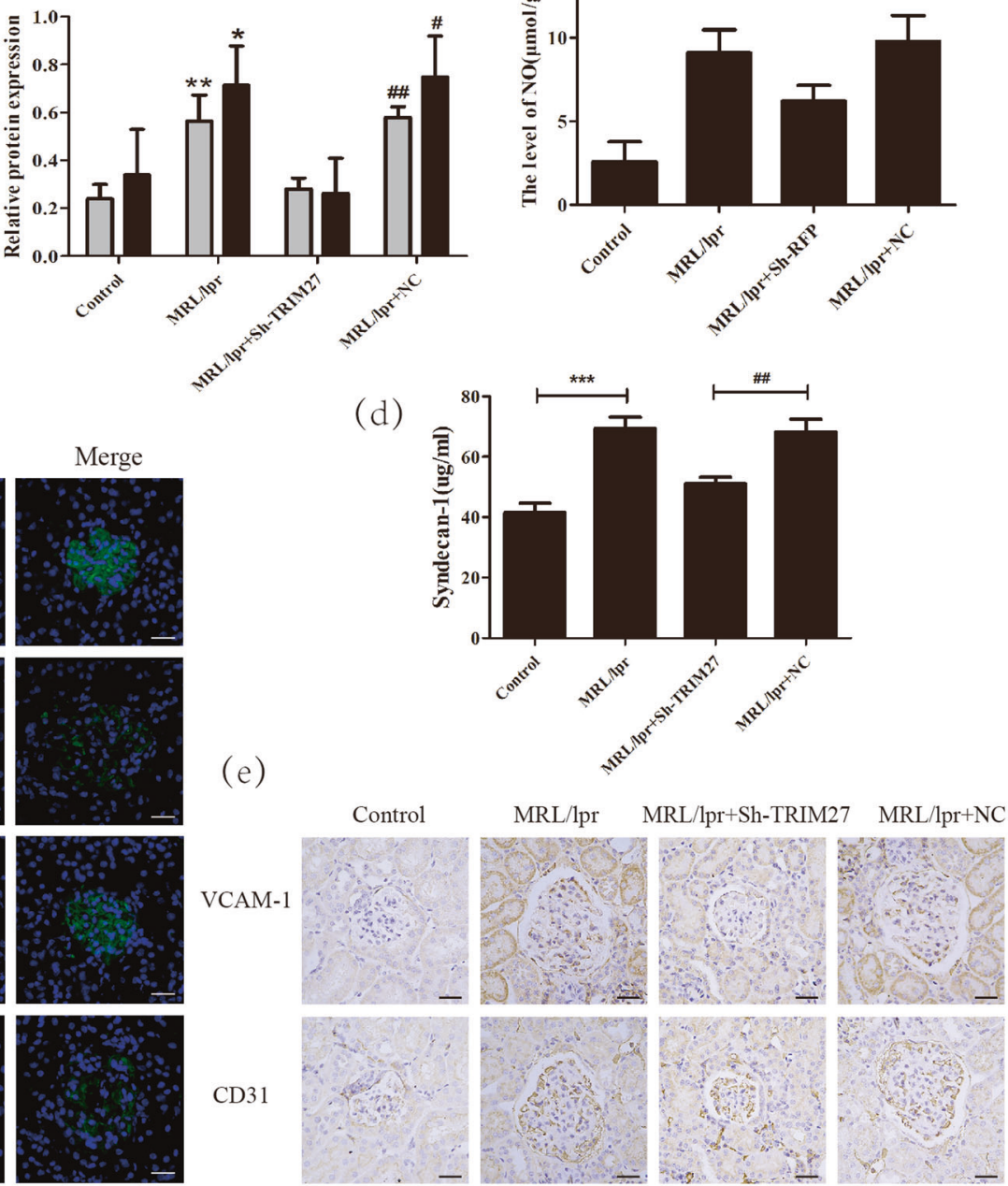

serum of mice in different groups. e IHC staining demonstrated the expression of VCAM-1 in the glomerular endothelial cells of mice. g The level of NO in the kidneys of mice in different groups. Scale bars: $50 \mu \mathrm{m} .{ }^{*} p<0.05,{ }^{* *} p<0.01,{ }^{* * *} p<0.001$ vs. control group, ${ }^{\#} p<$ $0.05,{ }^{\#} p<0.01,{ }^{\# \#} p<0.001$ vs. MRL/lpr + NC-AAV mice. TRIM27 tripartite motif-containing 27, IF immunofluorescence, ELISA enzyme-linked immunosorbent assay, IHC immunohistochemistry, VCAM-1 vascular cell adhesion molecule-1, NO nitric oxide. 


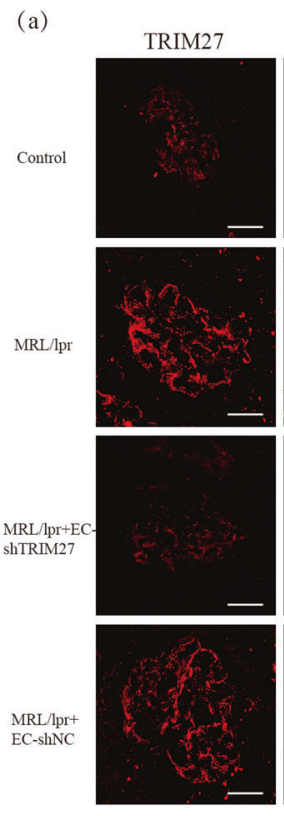

(c)
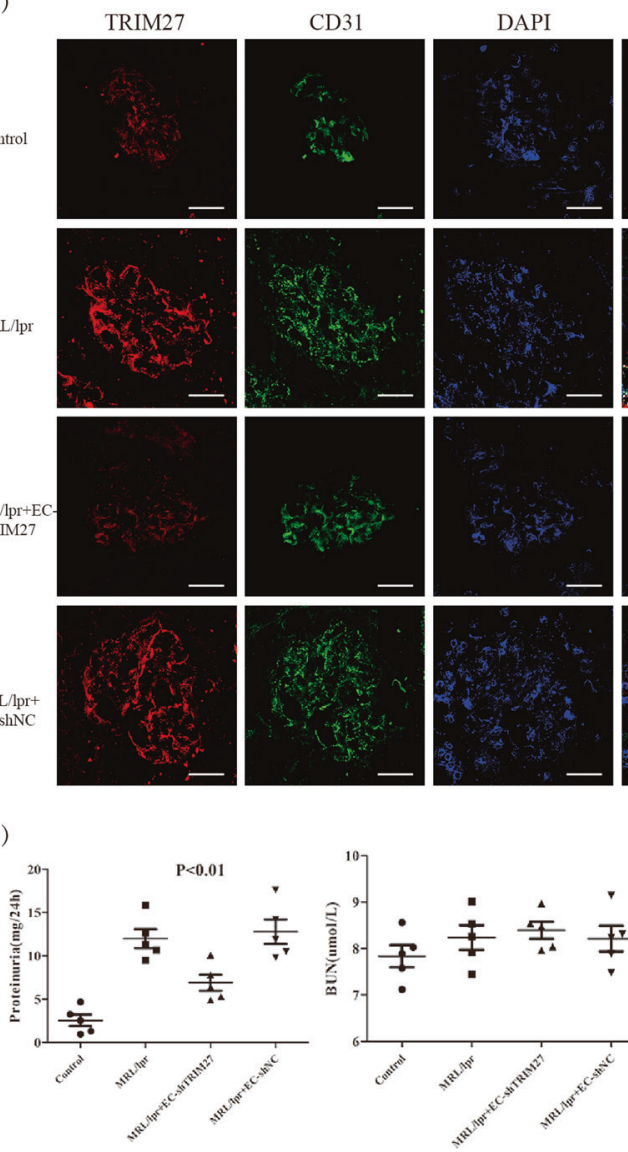

(d)
HE

HE

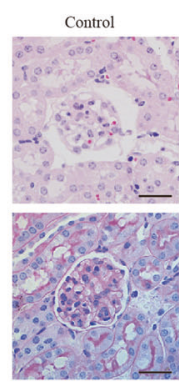

(g)

VCAM-1
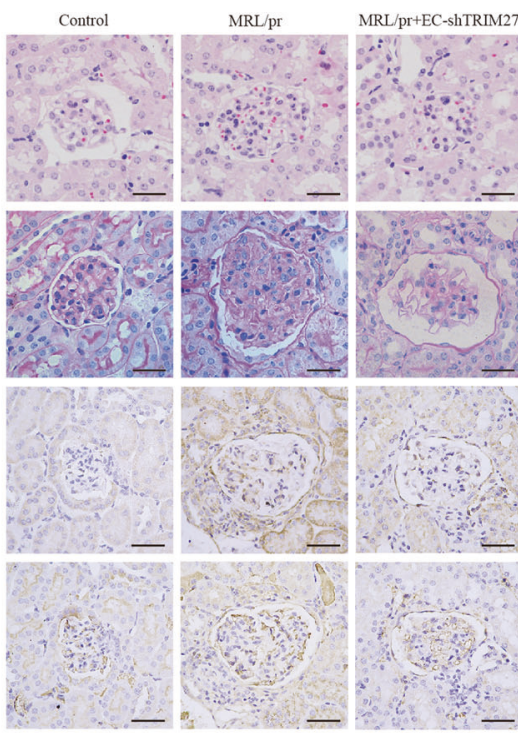

Fig. 2 Downregulation of TRIM27 expression inhibited the loss of the glycocalyx and glomerular endothelial cell injury in vivo. a, b IF and western blot analysis assay showed the expression of TRIM27 in glomerular endothelial cells was downregulated effectively in MRL/lpr+EC-shTRIM27-AAV group. c MRL/lpr mice injected with EC-shTRIM27-AAV showed less UPro/24 h than the NC-AAV group with little difference in Scr and BUN. d Representative HE and PASstained kidney sections from 24 weeks old mice after injected with ECshTRIM27-AAV or NC-AAV. e IF was applied to test the expression of syndecan-1 in the glomeruli of mice. $\mathbf{f}, \mathbf{h}$ The content of syndecan-1 and (b)

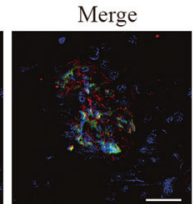

TRIM27 $-m-m$

VCAM-1 $=-\cdots-\cdots$

GAPDH - -
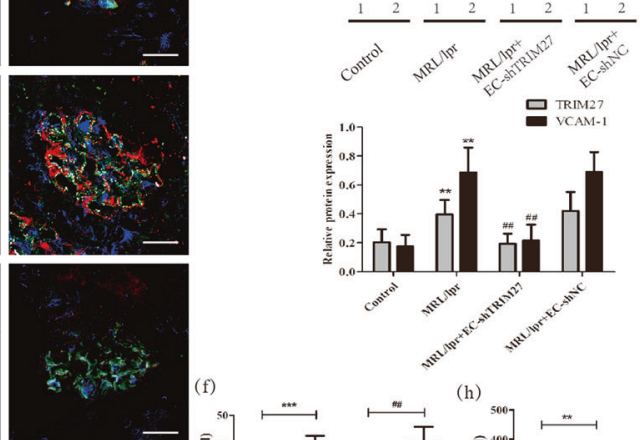

(f)

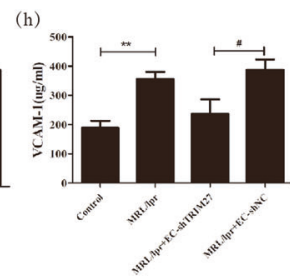

(i)
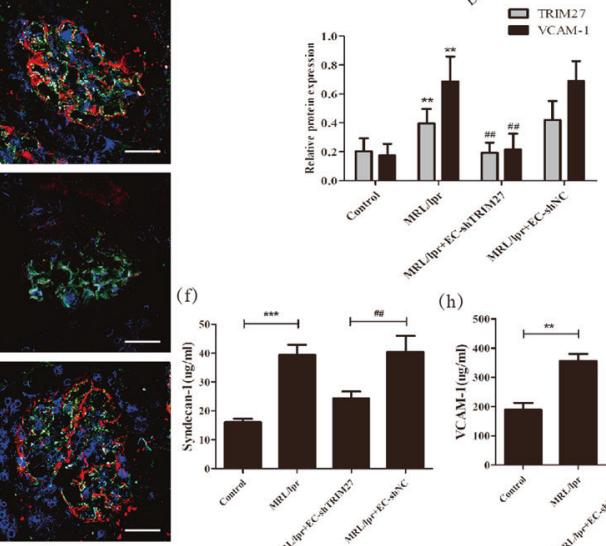
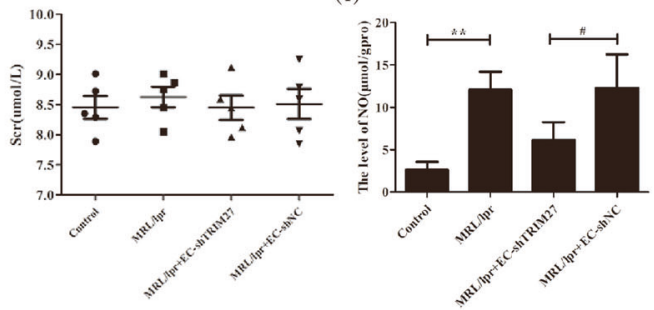

(e)
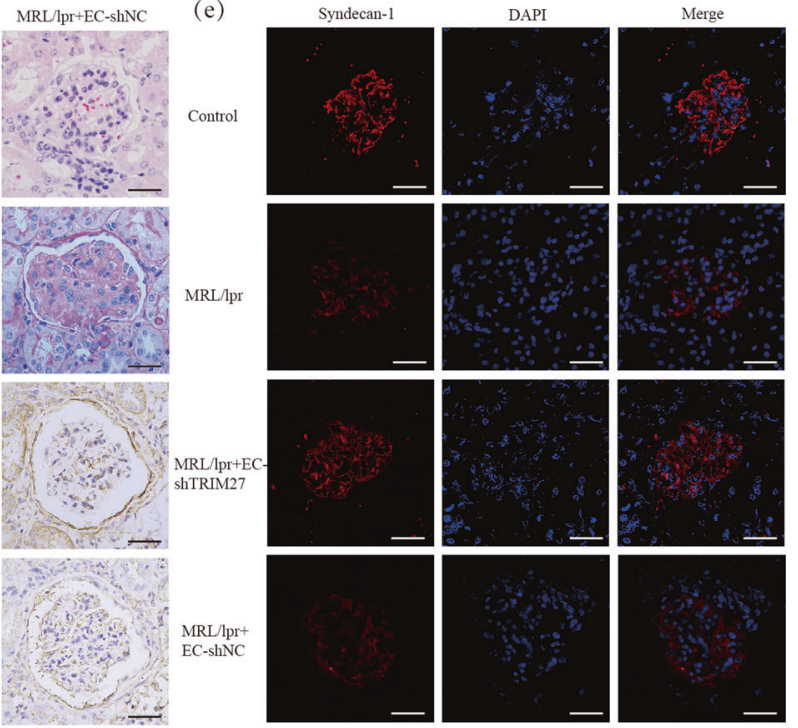

VCAM-1 in the serum of mice was measured by ELISA. $g$ The expression of VCAM-1 in the glomerular endothelial cells of mice was detected by IHC staining. $\mathbf{i}$ The level of NO in the kidneys of mice in different groups. Scale bars: $50 \mu \mathrm{m} .{ }^{*} p<0.05,{ }^{* *} p<0.01,{ }^{* * *} p<0.001$ vs. control group, ${ }^{\#} p<0.05,{ }^{\# \#} p<0.01,{ }^{\# \# \#} p<0.001$ vs. MRL/lpr + NCAAV mice. TRIM27 tripartite motif-containing 27, EC endothelial cell, IF immunofluorescence, Scr serum creatinine, BUN blood urea nitrogen, HE hematoxylin and eosin, PAS periodic acid-Schiff, ELISA enzyme-linked immunosorbent assay, IHC immunohistochemistry, VCAM-1 vascular cell adhesion molecule-1, NO nitric oxide. 

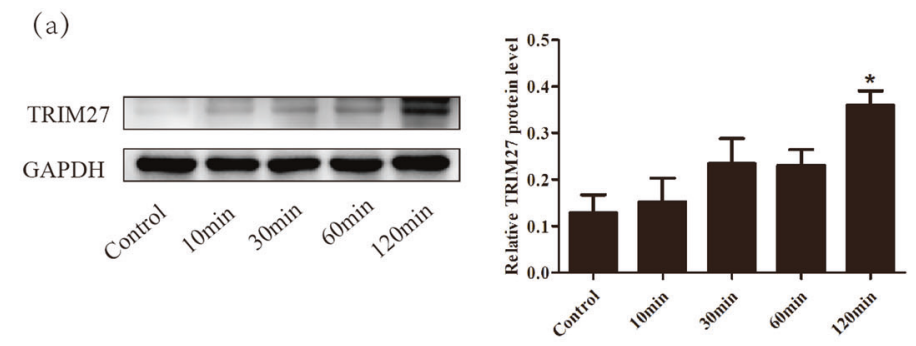

(h)
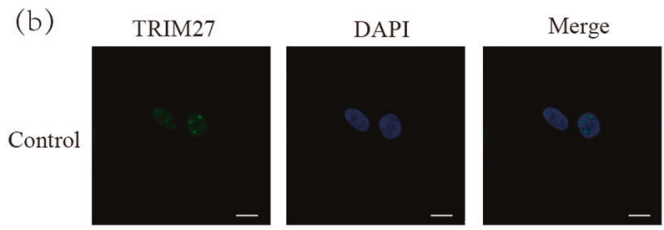

(c)
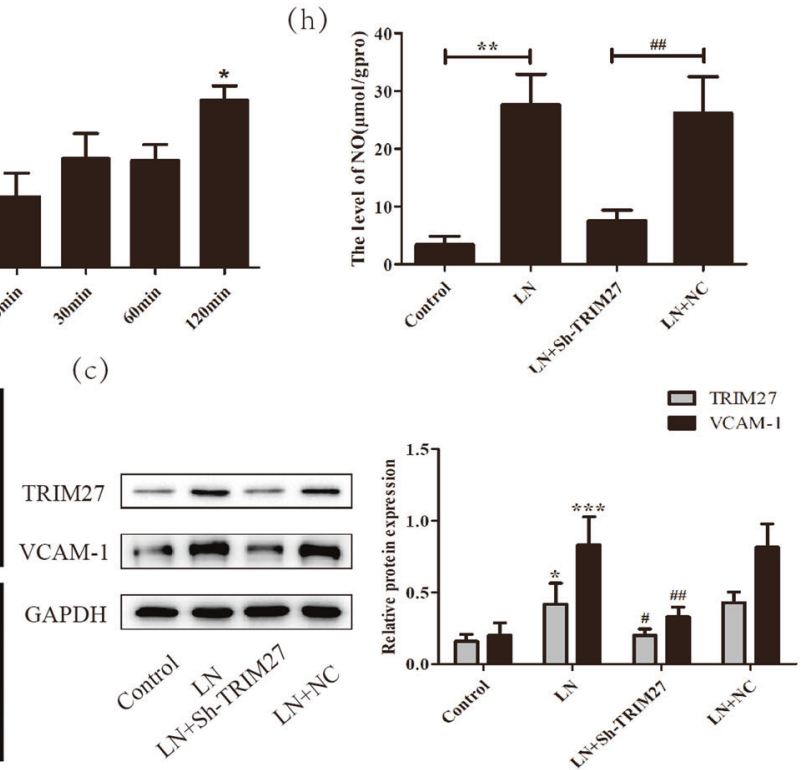
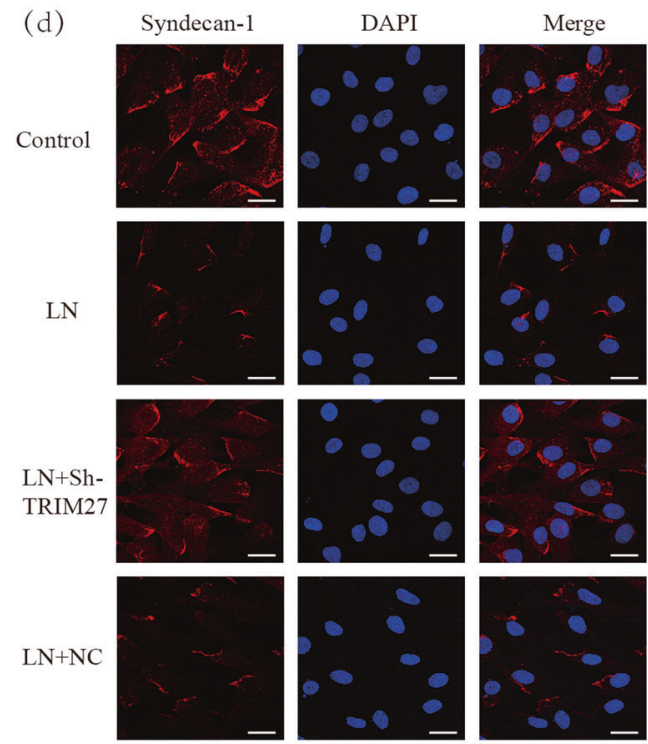

(e)

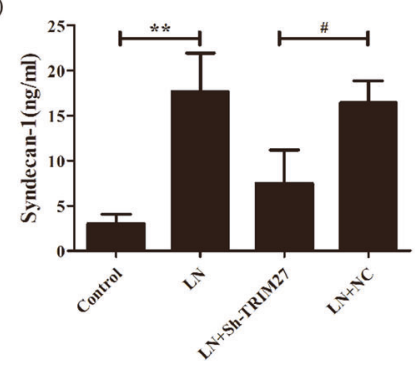

Fig. 3 Downregulation of TRIM27 reduced the injury of HRGECs induced by LN plasma. a Western blotting data showed that the expression of TRIM27 was higher after LN plasma stimulation in HRGECs than that in the control group. b IF results revealed that the expression of TRIM27 in HRGECs was greatly elevated in the cytoplasm after treatment with LN plasma. c Western blot analysis showed that the expression of TRIM27 and VCAM-1 were significantly reduced by infection of HRGECs with the sh-TRIM27 lentivirus. d Representative confocal microscopy images showing the expression of syndecan-1 in HRGECs. e, f Detection of the syndecan-1 and
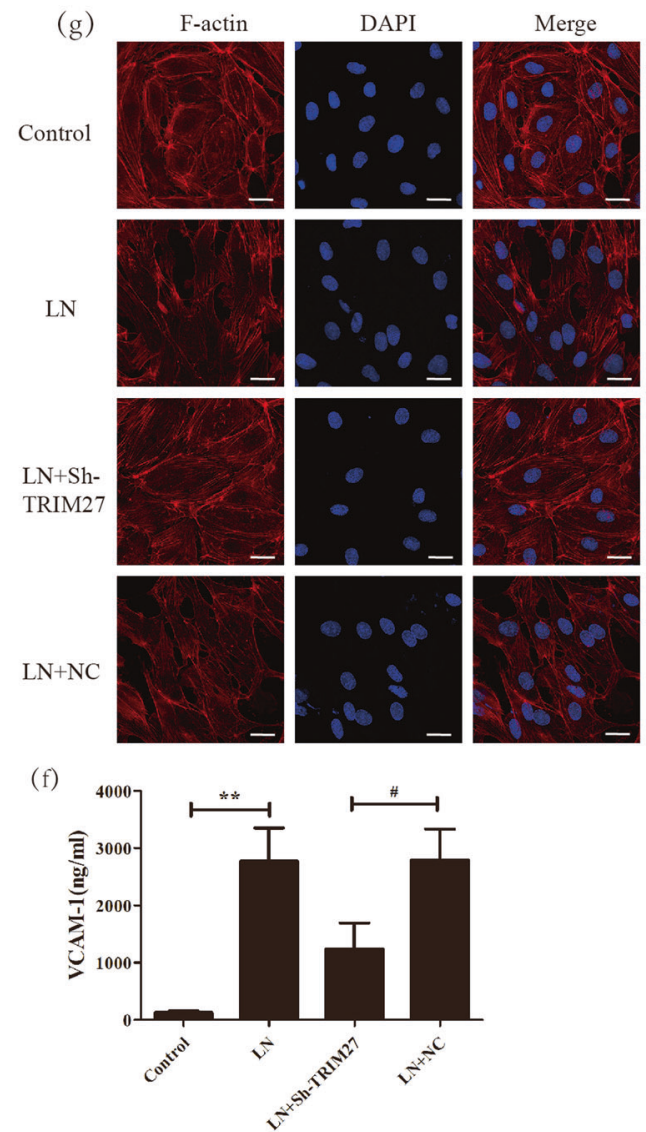

VCAM-1 proteins in the supernatant of HRGECs by ELISA. $\mathbf{g}$ IF was used to test the F-actin distribution of HRGECs in different groups. $\mathbf{h}$ Summarized data showing the level of NO in HRGECs with different treatments. Scale bars: $25 \mu \mathrm{m} .{ }^{*} p<0.05,{ }^{* *} p<0.01,{ }^{* * *} p<0.001$ vs. control group, ${ }^{\#} p<0.05,{ }^{\# \#} p<0.01$ vs. LN + NC group. TRIM27 tripartite motif-containing 27, HRGECs human renal glomerular endothelial cells, LN lupus nephritis, IF immunofluorescence, ELISA enzyme-linked immunosorbent assay, VCAM-1 vascular cell adhesion molecule-1, NO nitric oxide. 
protein level was dramatically enhanced in the LN group compared with that in the control group, while inhibition of TRIM27 expression suppressed the TRIM27 level. The ELISA result (Fig. 3f) for VCAM-1 was similar to that for syndecan-1. In addition, western blot analysis (Fig. 3c) showed that VCAM-1 protein expression was evidently increased after LN plasma stimulation compared with that in the control group, whereas transfection with TRIM27-shRNA-LV downregulated VCAM-1 protein expression.

The cytoskeleton of endothelial cells is critical for their function, so we used phalloidin to label F-actin. As shown in Fig. 3g, F-actin was regularly distributed across the whole cell in the control group. After treatment with LN plasma, the number of organized F-actin fibers was obviously decreased, whereas the number of disorganized F-actin fibers was evidently increased, and the fibers were arranged in an irregular way. However, the disordered arrangement of the cytoskeleton was partially ameliorated by TRIM27 knockdown.

Similarly, the level of NO in the HRGECs was tested. As shown in Fig. 3h, it was clearly increased after LN plasma treatment compared with that in the control group. Knockdown of the expression of TRIM27 significantly reduced the NO level, whereas little change in the NO level was observed in the NC-LV group.

\section{TRIM27 contributed to glomerular endothelial cell injury in lupus nephritis via the Fox01 pathway}

Previously, activation of the FoxO1 signaling pathway contributed to the TRIM27-mediated proliferation and extracellular matrix deposition of mesangial cells, and knockdown of TRIM27 decreased the level of proteinuria in LN mice [13]. To further investigate the precise effect of the FoxO1 pathway on the development of proteinuria, we analyzed the relationship between the activation of the FoxO1 signaling pathway and the dysfunction of glomerular endothelial cells. As shown in Fig. 4a, the protein expression of p-FoxO1-Ser256 was evidently increased at $30 \mathrm{~min}$ after $\mathrm{LN}$ plasma treatment, which was followed by a decrease in FoxO1 after $1 \mathrm{~h}$, which indicated that LN plasma could activate FoxO1 signaling in HRGECs.

To observe the effect of TRIM27 inhibition on the FoxO1 pathway, TRIM27-shRNA-LV or NC-LV was transfected into HRGECs. RT-PCR indicated that the mRNA level of FoxO1 was significantly increased after downregulation of TRIM27 (Fig. 4b). Western blotting results (Fig. 4c) revealed that after TRIM27 inhibition, the expression of p-FoxO1-Ser256 was remarkably reduced, which was accompanied by the elevation of FoxO1. Meanwhile, co-IP results showed that the LN plasma treatment could enhance the ubiquitination level of FoxO1, which was reduced by TRIM27 suppression (Fig. 4d). Notely, overexpression of TRIM27 could induce the reverse results (Fig. 4e-g).

Next, the influence of the overexpression of FoxO1 on the loss of the glycocalyx and glomerular endothelial cell injury was detected in vitro. Figure $5 \mathrm{a}$ demonstrates that the WT-FoxO1 vector effectively upregulated the expression of FoxO1 while producing little change in the $\mathrm{NC}$ group. The IF results (Fig. 5b) showed that the positive staining of the syndecan-1 protein was dramatically reduced in the LN group compared to that in the control group, which was consistent with the previous results. However, overexpression of FoxO1 partially recovered the expression of syndecan-1. The ELISA results (Figs. 5c, d) indicated that the WT-FoxO1 vector prohibited the upregulation of the syndecan-1 and VCAM-1 proteins in the medium, which were separately induced by LN plasma. In addition, the overexpression of FoxO1 reversed the effect of LN plasma on VCAM-1 protein expression (Fig. 5a).

Subsequently, as shown in Fig. 5b, the irregular arrangement of F-actin fibers was induced by LN plasma stimulation, which was partially diminished by transfection of the WT-FoxO1 vector.

Ultimately, the NO content was apparently increased in the LN plasma stimulation group compared with that in the control group, while transfection of the WT-FoxO1 vector reduced the NO content, which was in line with the expected results (Fig. 5e).

Taken together, these data demonstrated that the FoxO1 pathway contributed to glomerular endothelial cell injury mediated by TRIM27 in lupus nephritis.

\section{Active Akt upregulated the expression of the TRIM27 protein and then led to glomerular endothelial cell injury in lupus nephritis}

As active Akt could phosphorylate FoxO1, we explored its expression using western blot analysis. Figure 6a shows that the protein levels of p-Akt-Ser473 and p-Akt-Thr308 were dramatically enhanced at $30 \mathrm{~min}$ after LN plasma stimulation, whereas there was little change in Akt, indicating that LN plasma could activate the Akt signaling pathway. Thus, LY294002 was applied to inhibit the activation of Akt. As illustrated in Fig. 6b, the expression of p-Akt-Ser473 and pAkt-Thr308 were significantly decreased after LY294002 treatment.

Afterward, the effect of LY294002 on the expression of the TRIM27 and FoxO1 proteins was measured. As shown in Fig. 6c, LN plasma could increase the protein level of TRIM27, which was partially inhibited by LY294002. As expected (Fig. 6d), the inhibition of Akt activation effectively suppressed the phosphorylation of the FoxO1 protein, 
(a)

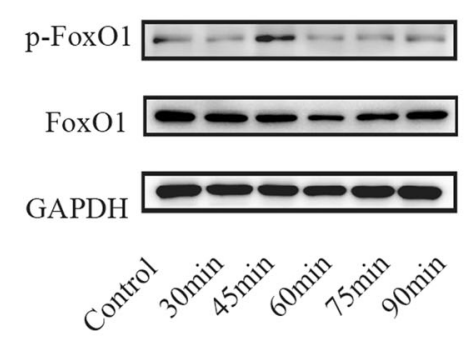

(b)

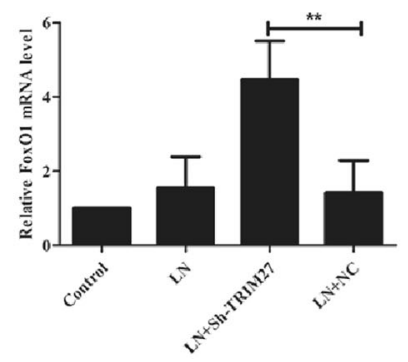

(e)

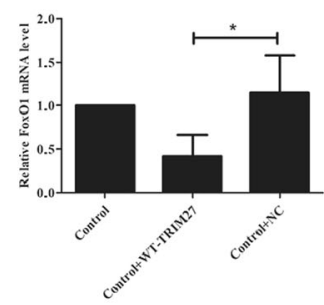

(f)

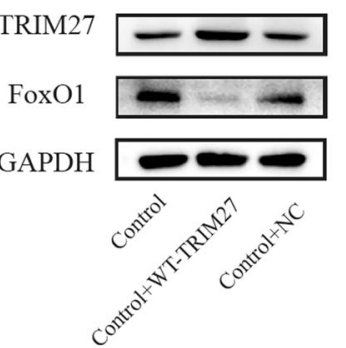

Fig. 4 TRIM27 participated in the loss of the glycocalyx and damage to HRGECs via the FoxO1 pathway. a Western blot analysis showed the expression of p-FoxO1-Ser256 and FoxO1 protein in HRGECs treated with LN plasma. b, c, e, f) Representative mRNA and protein level of TRIM27 and FoxO1 were detected in HRGECs after downregulate and upregulate the expression of TRIM27 separately by

which was accompanied by the upregulation of FoxO1. To verify whether Akt was the upstream molecule of TRIM27, the expression of TRIM27 protein was tested after treatment with SC79 (an agonist of Akt). The results demonstrated that activation of the Akt signaling pathway could increased the protein level of TRIM27 significantly (Fig. 6k), suggesting that TRIM27 was indeed the downstream molecule of Akt.

Then, we detected the influence of LY294002 on the dysfunction of endothelial cells treated with LN plasma by IF, western blotting, and ELISA methods. As expected, the results (Fig. 6e-j) demonstrated that suppression of Akt activation could reduce the impairment of the glycocalyx and the damage to endothelial cells.

These data revealed that activation of the Akt pathway contributed to glomerular endothelial cell injury via the TRIM27/FoxO1 signaling pathway in lupus nephritis. $\square \mathrm{p}-\mathrm{FoxOl}$

FoxOl

(d)
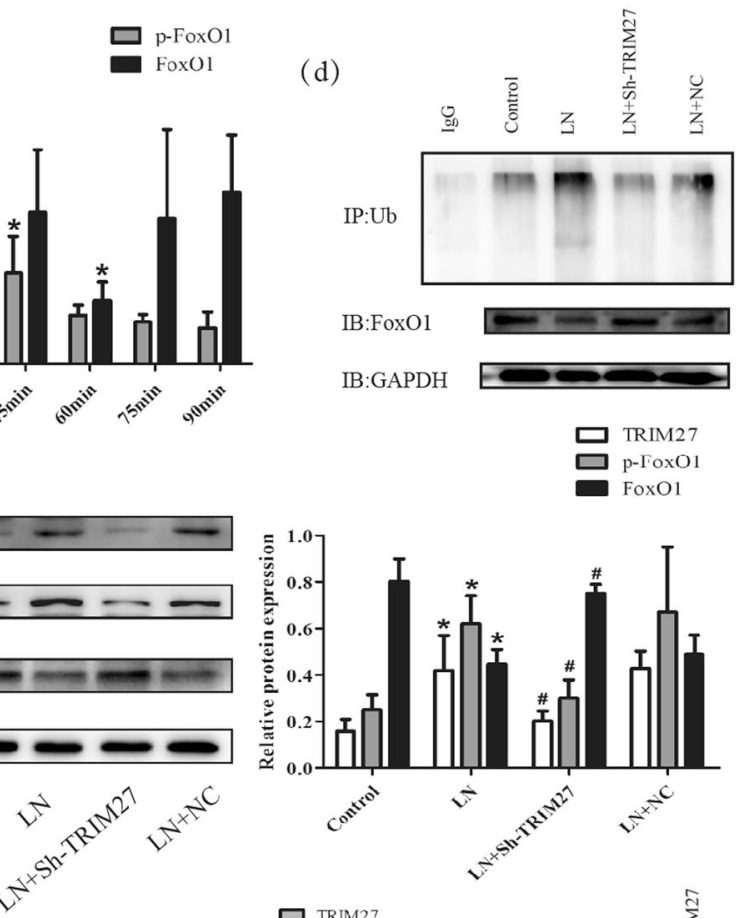

ㅁ TRIM27

믄이

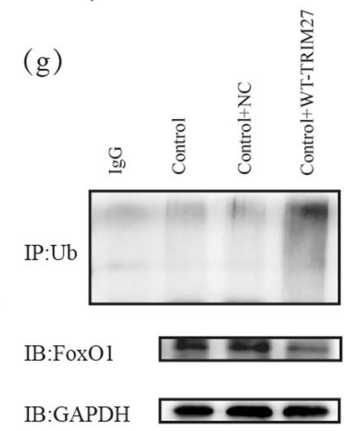

RT-PCR and western blot. $\mathbf{d}, \mathbf{g}$ The ubiquitination level of FoxO1 was tested by co-IP. ${ }^{*} p<0.05,{ }^{* *} p<0.01,{ }^{* * * *} p<0.001$ vs. control group, ${ }^{\#} p$ $<0.05,{ }^{\# \#} p<0.01$ vs. $\mathrm{LN}+\mathrm{NC}$ group. TRIM27 tripartite motifcontaining 27, HRGECs human renal glomerular endothelial cells, LN lupus nephritis, RT-PCR reverse transcription-polymerase chain reaction, co-IP co-immunoprecipitation.

\section{Discussion}

Previous studies have shown that the glycocalyx is a layer covering the surface of both fenestrated and nonfenestrated endothelial cells [18, 19]. The endothelial glycocalyx consists of proteoglycans, glycosaminoglycans, glycoproteins, and other soluble components [20]. Under physiological conditions, the degradation and regeneration of glycocalyx components are maintained in a dynamic balanced state. Increasing evidence has highlighted that the endothelial surface layer can be damaged when exposed to various stimuli, including shear stress, inflammatory cytokines, and oxidative stress, which may lead to several disorders, such as atherosclerosis, Takotsubo cardiomyopathy, and acute respiratory distress syndrome [21-23]. 

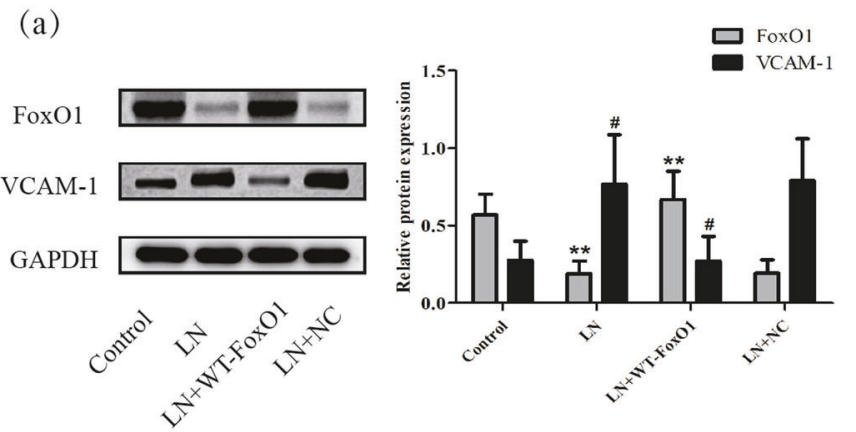

(b)

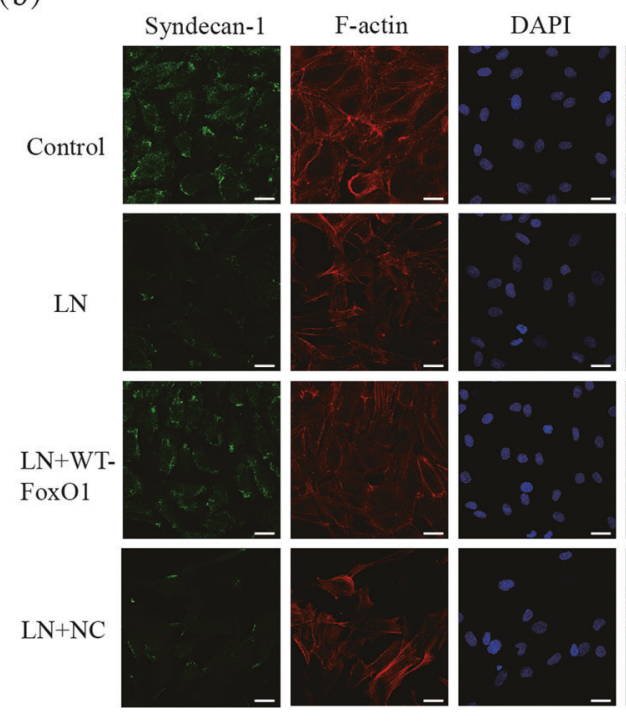

Fig. 5 Overepression of FoxO1 reduced the shedding of the glycocalyx and the damage of HRGECs in vitro. a Western blot analysis showed that the expression of FoxO1 was increased greatly and that of VCAM-1 was decreased significantly in HRGECs after transfection with the WT-FoxO1 plasmid. b IF detection showed the expression of SDC-1 and F-actin in HRGECs after different treatments. c, d Overexpression of FoxO1 repressed the levels of syndecan1 and VCAM-1 in the supernatant, as shown by ELISA. e The NO

In glomerular capillaries, the glycocalyx lines the fenestrated glomerular endothelial cells, which are a component of the glomerular filter barrier. Recently, increasing evidence has indicated that endothelial glycocalyx disruption plays an important role in a variety of kidney diseases. In chronic kidney disease patients (CKD) [24], the levels of syndecan-1 and hyaluronan (glycocalyx components) were increased significantly compared with those in healthy controls, which was correlated with the dysfunction of endothelial cells. In diabetic nephropathy $[25,26]$, the thickness of the endothelial glycocalyx was apparently reduced in mice injected with STZ. After restoring the endothelial glycocalyx [27], the level of albuminuria was remarkably reduced in diabetic mice. In addition, aldosterone, dysfunctional high-density lipoprotein, and uric acid could lead to the shedding of the glycocalyx and injury to endothelial cells [28-30]. $\mathrm{O}$ class 1. (c)

(d)

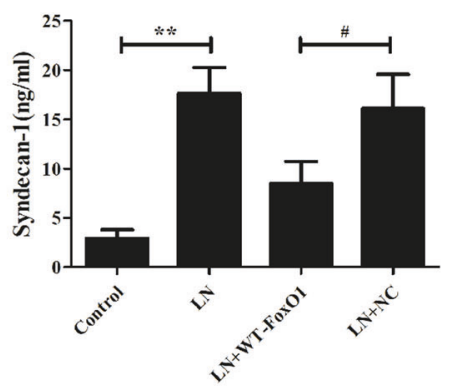

e)
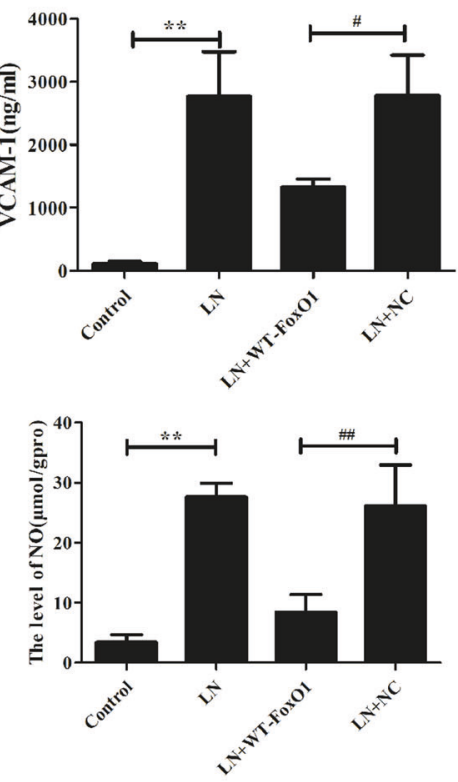

content in HRGECs with different treatments was measured. Scale bars: $25 \mu \mathrm{m} .{ }^{*} p<0.05,{ }^{* *} p<0.01$ vs. control group, ${ }^{\#} p<0.05,{ }^{\#} p<$ 0.01 vs. $\mathrm{LN}+\mathrm{NC}$ group. TRIM27 tripartite motif-containing 27, HRGECs human renal glomerular endothelial cells, LN lupus nephritis, IF immunofluorescence, ELISA enzyme-linked immunosorbent assay, VCAM-1 vascular cell adhesion molecule-1, NO nitric oxide, SDC-1 syndecan-1, FoxO1 forkhead transcription factors of the

As is the case for lupus nephritis, there has been little research about the endothelial glycocalyx. Kim KJ reported that serum syndecan-1 levels were higher in $14 \mathrm{LN}$ patients than those in healthy controls, which was closely related to the renal activity index [31]. In this study, we showed that the expression of the syndecan-1 protein in the glomerular cells of MRL/lpr mice was evidently reduced and accompanied by an increase in syndecan-1 in serum, which indicated impairment of the glycocalyx in LN mice. In addition, syndecan-1 protein expression in HRGECs was decreased, while in culture medium, it was elevated independently after stimulation with LN plasma. These data demonstrated the shedding of the endothelial glycocalyx in lupus nephritis.

Next, other experiments were employed to further detect endothelial cell injury in LN. The levels of serum VCAM-1 and NO have been reported to be increased in LN patients compared with those in healthy donors and are related to the 
Fig. 6 The activation of Akt induced the abnormal functioning of HRGECs through the TRIM27/FoxO1 pathway in vitro.

a The expression of p-AktSer473, p-Akt-Thr308 and Akt were detected by western blotting in HRGECs. $\mathbf{b}$ The efficiency of LY294002 was measured by western blotting.

c, d Western blot analysis showed the expression of TRIM27, p-FoxO1-Ser256 and FoxO1 protein after LY294002 pretreatment in HRGECs. e IF was used to detect the expression of syndecan-1 after using LY294002 in HRGECs.

f, h ELISA showed that LY294002 could reduce the expression of syndecan-1 and VCAM-1 in the supernatant. g Western blot analysis showed that VCAM-1 protein expression decreased significantly after LY294002 pretreatment in HRGECs. (i) IF detection of the distribution of F-actin after stimulation with LY294002 of HRGECs. $\mathbf{j}$ The results showed that the level of NO decreased significantly after pretreatment with LY294002 in HRGECs.

k Western blot analysis showed that TRIM27 protein expression increased dramatically after SC79 pretreatment in HRGECs. Scale bars: $25 \mu \mathrm{m} .{ }^{*} p<0.05$, ${ }^{* *} p<0.01$ vs. control group, ${ }^{\#} p<0.05,{ }^{\# \#} p<0.01$ vs. LN + DM group. TRIM27 tripartite motif-containing 27, HRGECs human renal glomerular endothelial cells, $\mathrm{LN}$ lupus nephritis, IF immunofluorescence, ELISA enzyme-linked immunosorbent assay, VCAM-1 vascular cell adhesion molecule1, NO nitric oxide, LY LY294002, DM DMSO, SC SC79.
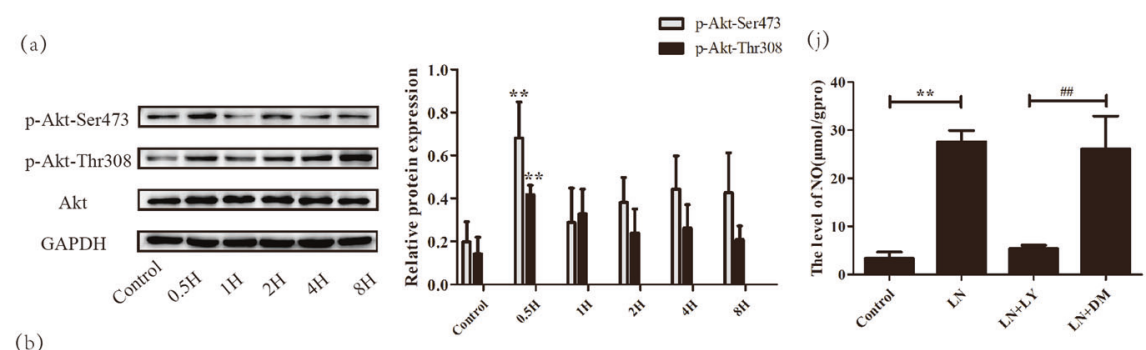

(b)

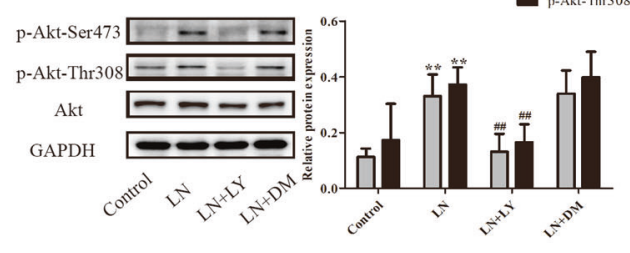

(c)
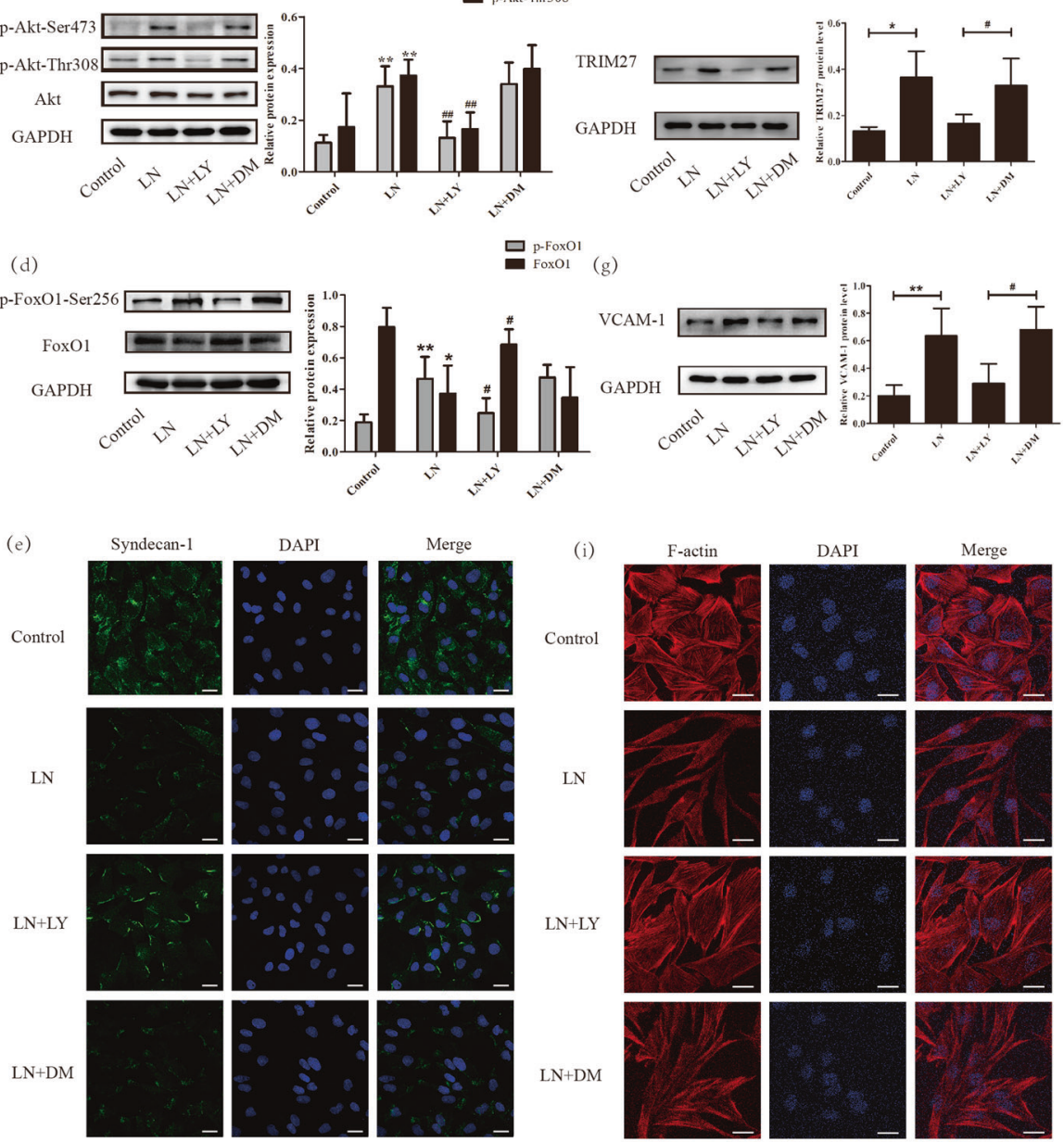

(f)
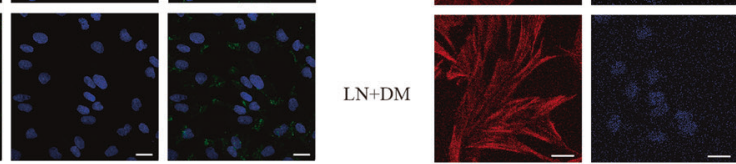

(k)
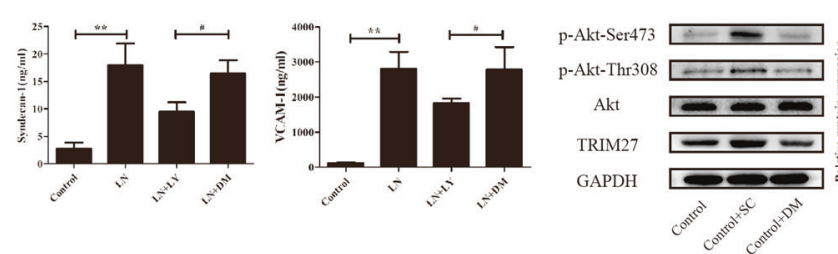

믐 $\begin{gathered}\text { p-Akt-Ser473 } \\ \text { p-Akt-Phrs08 }\end{gathered}$
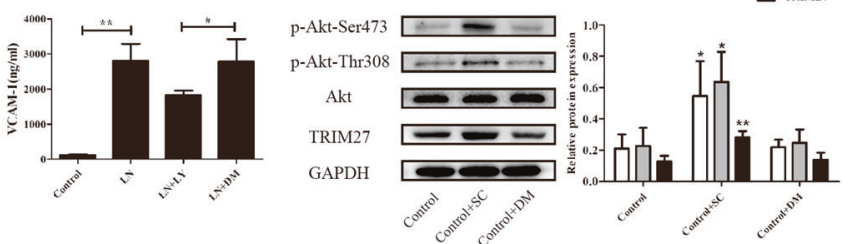

damage of endothelial cells [32-36]. Therefore, we tested the expression of VCAM-1 protein and NO production in vivo and in vitro. Expectedly, the results showed that both were upregulated in the glomerular cells of LN mice and HRGECs treated with LN plasma, which was consistent with previous reports. In addition, phalloidin staining demonstrated the disordered arrangement of F-actin protein in LN plasma-stimulated HRGECs, which also indicated the occurrence of endothelial cell injury.

Previous research has reported that TRIM27 is involved in various biological actions, including mediation of signaling pathways, transcriptional regulation, cell differentiation, and the inflammatory response [37-40]. Recently, it has been shown that overexpression of TRIM27 plays a role 
in colon, esophageal, and intraductal carcinoma [41-43]. In a previous study, we revealed that the TRIM27 protein was highly expressed in the glomerular mesangial cells of LN patients and mice, where it mediated mesangial cell proliferation and extracellular matrix deposition [13]. Interestingly, during the experiments, TRIM27 was also found to be upregulated in the glomerular endothelial cells of lupus nephritis. In addition, other studies have shown that TRIM14 and TRIM28 promote endothelial activation $[44,45]$. Does TRIM27 play a role in the breakdown of the glycocalyx and injury to endothelial cells? The results demonstrated that downregulation of TRIM27 could partially preserve the glycocalyx and reduce the damage of endothelial cells, which was consistent with previous research. This effect may be due to the structural similarity, which needs to be further investigated.

Therefore, the way in which TRIM27 mediates the dysfunction of endothelial cells in LN was explored. Shen B discovered that silencing FoxO1 could suppress endothelial cell injury [46]. Bertin FR showed that knockdown of FoxO1 enhanced the expression of VCAM-1 in endothelial cells [47]. Our study revealed that TRIM27 could regulate the proliferation of mesangial cells via the FoxO1/p27 pathway [13]. Therefore, whether the TRIM27/ FoxO1 signaling pathway is involved in endothelial cell injury was investigated. The results indicated that suppression of TRIM27 could prevent FoxO1 expression and that the upregulation of FoxO1 attenuated the loss of the endothelial cell surface layer and damage to endothelial cells in LN. However, the mechanism by which TRIM27 mediates the expression of FoxO1 remains unclear. Ranjit [48] reported that TRIM27 inhibition increased the activity of cis-regulatory elements located in the FoxO1 gene, which was consistent with our results that TRIM27 could regulate the mRNA level of FoxO1. Besides, we found the ubiquitination level of FoxO1was modulated by TRIM27 which may be related with the ubiquitin ligase activity of TRIM 27 and will be further explored in the future.

Other research teams have highlighted that Akt, an upstream regulator of FoxO1, influences the dysfunction of endothelial cells. In diabetic nephropathy, an increase in endoglin contributes to the activation of endothelial cells by regulating Akt signaling [49]. Bevan HS showed that activation of the PI3K/Akt pathway increased the production of NO induced by laminar shear stress in endothelial cells [50]. In this study, LN plasma stimulation activated PI3K/Akt signaling in HRGECs. Importantly, inhibiting the Akt pathway with LY294002 significantly reduced the protein level of TRIM27 and the upregulation of the FoxO1 protein, which restored the glycocalyx and ameliorated the injury of HRGECs. Meanwhile, the expression of TRIM27 protein was significantly enhanced after activation of Akt with SC79 which further confirmed Akt was the upstream molecule of TRIM27. These results were distinct from the research that have been reported by Ma [51], which may be caused by the different microenvironment of cells and dissimilar pathogenesis of $\mathrm{LN}$ and cancer.

Taken together, our study revealed that TRIM27 played a precise role in the dysfunction of endothelial cells in lupus nephritis. Downregulation of TRIM27 suppressed the impairment of the glycocalyx and the damage of endothelial cells by the FoxO1 pathway. Activation of Akt could upregulate the expression of TRIM27, which was revealed to be a novel regulator of the injury of endothelial cells in LN, providing a novel strategy for the treatment of lupus nephritis.

\section{Data availability}

The data that support the findings of this study are available from the corresponding author upon reasonable request.

Acknowledgements This work was supported by the National Natural Science Foundation of China (81970600), Natural Science Foundation of Hebei Province (H2020206195), and the Department of Education of Hebei (ZD2018006, QN2021099).

Author contributions XF and SL designed the study and helped to revise the manuscript. JL and JX analyzed the data, wrote and edited the manuscript. JH and CG performed the experiments in vitro. QL and WZ contributed with their expertize in medical issues and helped edit the manuscript. FG made the paraffin section of the kidney. YT, $\mathrm{XM}, \mathrm{ZZ}, \mathrm{BJ}, \mathrm{YT}, \mathrm{LW}$, and $\mathrm{HZ}$ completed the experiments in vivo including western blot analysis, IF, ELISA, Real-time PCR, CO-IP. All authors have read and approved the final version of the manuscript, and agreed with the order of presentation of the authors.

Funding This work was supported by the National Natural Science Foundation of China (81970600) and the Department of Education of Hebei (ZD2018006).

\section{Compliance with ethical standards}

Conflict of interest The authors declare no competing interests.

Ethics approval The study was approved by the Clinical Research Ethics Committee of the Second Hospital of Hebei Medical University (20190017). Written informed consent was obtained from each study participant.

Publisher's note Springer Nature remains neutral with regard to jurisdictional claims in published maps and institutional affiliations.

\section{References}

1. Parikh SV, Almaani S, Brodsky S, Rovin BH. Update on lupus nephritis: core curriculum 2020. Am J Kidney Dis. 2020;76:265-81.

2. Tektonidou MG, Dasgupta A, Ward MM. Risk of end-stage renal disease in patients with lupus nephritis, 1971-2015: a systematic review and bayesian meta-analysis. Arthritis Rheumatol. 2016;68:1432-41. 
3. Satchell S. The role of the glomerular endothelium in albumin handling. Nat Rev Nephro. 2013;19:717-25.

4. Li AS, Ingham JF, Lennon R. Genetic disorders of the glomerular filtration barrier. Clin J Am Soc Nephrol. 2020;15:1818-28.

5. Tisher, CC Functional anatomy of the kidney. Hosp Pract. 1978;13:53-65.

6. Patrakka J, Tryggvason K. Molecular make-up of the glomerular filtration barrier. Biochem Biophys Res Commun. 2010;396:164-9.

7. Haraldsson B, Nystrom J, Deen WM. Properties of the glomerular barrier and mechanisms of proteinuria. Physiol Rev. 2008;88:451-87.

8. Nawata A, Izumi R, Harada K, Kurisu H, Shimajiri S, Matsuki Y, et al. Podocyte and endothelial cell injury lead to nephrotic syndrome in proliferative lupus nephritis. Histopathology. 2018;72:1084-92.

9. Micale L, Chaignat E, Fusco C, Reymond A, Merla G. The tripartite motif: structure and function. Adv Exp Med Biol. 2012;770:11-25.

10. Jaworska AM, Wlodarczyk NA, Mackiewicz A, Czerwinska P. The role of TRIM family proteins in the regulation of cancer stem cell self-renewal. Stem Cells. 2020;38:165-73.

11. Van Gent M, Sparrer KMJ, Gack MU. TRIM proteins and their roles in antiviral host defenses. Annu Rev Virol. 2018;5:385-405.

12. Zheng $\mathrm{F}, \mathrm{Xu} \mathrm{N}$, Zhang Y. TRIM27 promotes hepatitis $\mathrm{C}$ virus replication by suppressing type I interferon response. Inflammation. 2019;42:1317-25.

13. Liu J, Feng X, Tian Y, Wang K, Gao F, Yang L, et al. Knockdown of TRIM27 expression suppresses the dysfunction of mesangial cells in lupus nephritis by FoxO1 pathway. J Cell Physiol. 2019;234:11555-66.

14. Shao Y, Cheng Z, Li X, Chernaya V, Wang H, Yang XF. Immunosuppressive/anti-inflammatory cytokines directly and indirectly inhibit endothelial dysfunction-a novel mechanism for maintaining vascular function. J Hematol Oncol. 2014;7:1-14.

15. Wuthrich RP. Vascular cell adhesion molecule-1 (VCAM-1) expression in murine lupus nephritis. Kidney Int. 1992;4:903-14.

16. Dellamea BS, Leitão CB, Friedman R, Canani LH. Nitric oxide system and diabetic nephropathy. Diabetol Metab Syndr. 2014;6:1-6.

17. Komers R, Anderson S. Paradoxes of nitric oxide in the diabetic kidney. Am J Physiol Renal Physiol. 2003;284:1121-37.

18. Liew H, Roberts MA, MacGinley R, McMahon LP. Endothelial glycocalyx in health and kidney disease: Rising star or false Dawn? Nephrology (Carlton). 2017;22:940-6.

19. Garsen M, Rops AL, Rabelink TJ, Berden JH, van der Vlag J. The role of heparanase and the endothelial glycocalyx in the development of proteinuria. Nephrol Dial Transplant. 2014;29:49-55.

20. Kolarova H, Ambruzova B, Svihalkova SL, Klinke L, Kubala L. Modulation of endothelial glycocalyx structure under inflammatory conditions. Mediators Inflamm. 2014;2014:694312.

21. Suzuki K, Okada H, Takemura G, Takada C, Tomita H, Yano H, et al. Recombinant thrombomodulin protects against LPS-induced acute respiratory distress syndrome via preservation of pulmonary endothelial glycocalyx. Br J Pharmacol. 2020;177:4021-33.

22. Mitra R, O'Neil GL, Harding IC, Cheng MJ, Mensah SA, Ebong EE. Glycocalyx in atherosclerosis-relevant endothelium function and as a therapeutic target. Curr Atheroscler Rep. 2017;19:63.

23. Nguyen TH, Liu S, Ong GJ, Stafford I, Frenneaux MP, Horowitz JD. Glycocalyx shedding is markedly increased during the acute phase of Takotsubo cardiomyopathy. Int J Cardiol. 2017;243:296-9.

24. Liew H, Roberts MA, Pope A, McMahon LP. Damage of the endothelial glycocalyx in chronic kidney disease. Atherosclerosis. 2014;234:335-43.

25. Oltean S, Qiu Y, Ferguson JK, Stevens M, Neal C, Russell A, et al. Vascular endothelial growth factor-A165b is protective and restores endothelial glycocalyx in diabetic nephropathy. J Am Soc Nephrol. 2015;26:1889-904.

26. Zhang Y, Ma KL, Gong YX, Wang GH, Hu ZB, Liu L, et al. Platelet microparticles mediate glomerular endothelial injury in early diabetic nephropathy. J Am Soc Nephrol. 2018;29:2671-95.

27. Boels MGS, Koudijs A, Avramut MC, Sol WMPJ, Wang G, van Oeveren-Rietdijk AM. Systemic monocyte chemotactic protein-1 inhibition modifies renal macrophages and restores glomerular endothelial glycocalyx and barrier function in diabetic nephropathy. Am J Pathol. 2017;187:2430-40.

28. Hesse B, Rovas A, Buscher K, Kusche-Vihrog K, Brand M, Di Marco GS, et al. Symmetric dimethylarginine in dysfunctional high-density lipoprotein mediates endothelial glycocalyx breakdown in chronic kidney disease. Kidney Int. 2020;97:502-15.

29. Ko J, Kang HJ, Kim DA, Kim MJ, Ryu ES, Lee S, et al. Uric acid induced the phenotype transition of vascular endothelial cells via induction of oxidative stress and glycocalyx shedding. FASEB J. 2019;33:13334-45.

30. Butler MJ, Ramnath R, Kadoya H, Desposito D, Riquier-Brison A, Ferguson JK, et al. Aldosterone induces albuminuria via matrix metalloproteinase-dependent damage of the endothelial glycocalyx. Kidney Int. 2019;95:94-107.

31. Kim KJ, Kim JY, Baek IW, Kim WU, Cho CS. Elevated serum levels of syndecan-1 are associated with renal involvement in patients with systemic lupus erythematosus. J Rheumatol. 2015;42:202-9.

32. Page AV, Liles WC. Biomarkers of endothelial activation/dysfunction in infectious diseases. Virulence. 2013;4:507-16.

33. Oates JC, Shaftman SR, Self SE, Gilkeson GS. Association of serum nitrate and nitrite levels with longitudinal assessments of disease activity and damage in systemic lupus erythematosus and lupus nephritis. Arthritis Rheum. 2008;58:263-72.

34. El-Magadmi M, Bodill H, Ahmad Y, Durrington PN, Mackness M, Walker M, et al. Systemic lupus erythematosus: an independent risk factor for endothelial dysfunction in women. Circulation. 2004;110:399-404.

35. Lewis MJ, Vyse S, Shields AM, Zou L, Khamashta M, Gordon $\mathrm{PA}$, et al. Improved monitoring of clinical response in Systemic Lupus Erythematosus by longitudinal trend in soluble vascular cell adhesion molecule-1. Arthritis Res Ther. 2016;18:5.

36. Da Rosa Franchi Santos LF, Stadtlober NP, Costa Dall'Aqua LG, Scavuzzi BM, Guimarães PM. Increased adhesion molecule levels in systemic lupus erythematosus: relationships with severity of illness, autoimmunity, metabolic syndrome and cortisol levels. Lupus. 2018;27:380-8.

37. Conwell SE, White AE, Harper JW, Knipe DM. Identification of TRIM27 as a novel degradation target of herpes simplex virus 1 ICP0. J Virol. 2015;89:220-9.

38. Lee JT, Shan J, Zhong J, Li M, Zhou B, Zhou A, et al. RFPmediated ubiquitination of PTEN modulates its effect on AKT activation. Cell Res. 2013;23:552-64.

39. Nie D, Zhang D, Dai J, Zhang M, Zhao X, Xu W, et al. Nicotine induced murine spermatozoa apoptosis via up-regulation of deubiquitinated RIP1 by Trim27 promoter hypomethylation. Biol Reprod. 2016;94:31.

40. Wang T, Xia C, Dong Y, Chen X, Wang J, Du J. Trim 27 confers myeloid hematopoiesis competitiveness by up-regulating myeloid master genes. J Leukoc Biol. 2018;104:799-809.

41. Ma L, Yao N, Chen P, Zhuang Z. TRIM27 promotes the development of esophagus cancer via regulating PTEN/AKT signaling pathway. Cancer Cell Int. 2019;19:283.

42. Zhang HX, Xu ZS, Lin H, Li M, Xia T, Cui K, et al. TRIM27 mediates STAT3 activation at retromer-positive structures to promote colitis and colitis-associated carcinogenesis. Nat Commun. 2018;9:3441.

43. Skálová A, Ptáková N, Santana T, Agaimy A, Ihrler S, Uro-Coste E, et al. NCOA4-RET and TRIM27-RET are characteristic gene fusions in salivary intraductal carcinoma, including invasive and metastatic tumors: is "intraductal" correct? Am J Surg Pathol. 2019;43:1303-13. 
44. Wang Y, Li J, Huang Y, Dai X, Liu Y, Liu Z, et al. Tripartite motif-containing 28 bridges endothelial inflammation and angiogenic activity by retaining expression of TNFR-1 and -2 and VEGFR2 in endothelial cells. FASEB J. 2017;31:2026-36.

45. Huang X, Li Y, Li X, Fan D, Xin HB, Fu M. TRIM14 promotes endothelial activation via activating NF-kappaB signaling pathway. J Mol Cell Biol. 2020;12:176-89.

46. Shen B, Gao L, Hsu YT, Bledsoe G, Hagiwara M, Chao L, et al. Kallistatin attenuates endothelial apoptosis through inhibition of oxidative stress and activation of Akt-eNOS signaling. Am J Physiol Heart Circ Physiol. 2010;299:H1419-27.

47. Bertin FR, Lemarié CA, Robins RS, Blostein MD. Growth arrestspecific 6 regulates thrombin-induced expression of vascular cell adhesion molecule-1 through forkhead box $\mathrm{O} 1$ in endothelial cells. J Thromb Haemost. 2015;13:2260-72.
48. Ranjit M, Hirano M, Aoki K, Okuno Y, Ohka F, Yamamichi A, et al. Aberrant active cis-regulatory elements associated with downregulation of RET finger protein overcome chemoresistance in glioblastoma. Cell Rep. 2019;26:2274-81. e2275.

49. Bus P, Gerrits T, Heemskerk SAC, Zandbergen M, Wolterbeek R, Bruijn JA, et al. Endoglin mediates vascular endothelial growth factor-A-induced endothelial cell activation by regulating Akt signaling. Am J Pathol. 2018;188:2924-35.

50. Bevan HS, Slater SC, Clarke H, Cahill PA, Mathieson PW, Welsh GI, et al. Acute laminar shear stress reversibly increases human glomerular endothelial cell permeability via activation of endothelial nitric oxide synthase. Am J Physiol Renal Physiol. 2011;301:F733-42.

51. Ma Y, Wei Z, Bast RC Jr, Wang Z, Li Y, Gao M, et al. Downregulation of TRIM27 expression inhibits the proliferation of ovarian cancer cells in vitro and in vivo. Lab Invest. 2016;96:37-48. 